\title{
The efficacy of strategies used to minimise or prevent Cisplatin-induced ototoxicity in patients
}

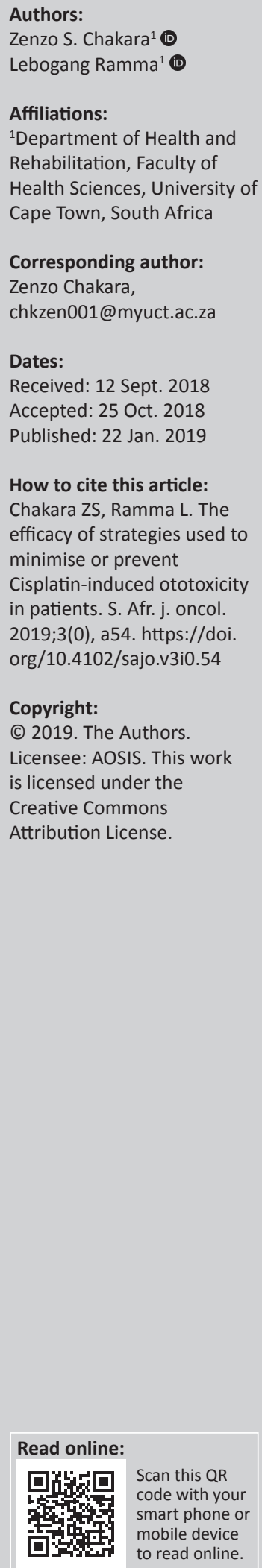

Background: Hearing loss is a major side effect of cisplatin-based chemotherapy. With a large burden of cancer in developing countries, an evidence-based approach to prevention of cisplatin-induced hearing loss (ototoxicity) is pertinent.

Aim: This study aimed to evaluate the efficacy of treatment modifications currently being implemented to prevent or minimise ototoxicity during cisplatin-based chemotherapy.

Setting: A retrospective medical folder review design was employed. Purposive sampling was used to select medical folders of adult patients who were undergoing cisplatin-based chemotherapy at Groote Schuur Hospital between January 2011 and December 2016.

Method: Demographic, cisplatin chemotherapy treatment and audiometric data were extracted from patients' records. The Common Terminology Criteria of Adverse Events version 4 (CTCAE v4) grading scale was used to determine ototoxicity and grade severity of hearing loss. Data were analysed using $\mathrm{R}$, a software environment for statistical computing.

Results: Fifty-eight medical folders were included in the study (median age $=43$ years; range: 18-75 years; 36 male, 22 female; average length of treatment: 13.45 weeks). Three treatment modifications were used: Dose reduction, switching drugs and continuing with the same drug. Common Terminology Criteria of Adverse Events version 4 grading scale revealed ototoxicity in $75 \%$ of the patients who switched drugs, $50 \%$ of the patients with reduced dose and $56 \%$ of the patients who were continued on the same drug. There was no statistically significant association between treatment modifications and incidence of ototoxicity.

Conclusion: Most patients experienced cisplatin-induced ototoxicity despite treatment modifications. There was no statistically significant association between any of the strategies implemented and incidence of hearing loss.

\section{Introduction}

Cancer is amongst the most dominant non-communicable diseases and claims more lives than the combination of human immunodeficiency virus and acquired immunodeficiency syndrome (HIV and AIDS), malaria and tuberculosis worldwide. ${ }^{1,2}$ According to the World Health Organization, ${ }^{3}$ 8.2 million deaths and 14 million new cases of cancer were recorded globally in 2012. In South Africa, approximately 100000 people are diagnosed with cancer annually, and numbers are expected to rise by $46 \%$ by $2030 .{ }^{4}$ With approximately $12 \%$ of the South African population being infected by HIV and AIDS and resultantly being exposed to antiretroviral treatment, the risk of developing HIV-related cancers, such as Kaposi's sarcoma, is likely to create a great burden on the already strained health system..$^{2,4}$

Survival rates for cancer are on the rise owing to development of more effective chemotherapeutic regimens. ${ }^{5,6}$ A paradoxical concern emerging from this breakthrough in health care is that affected individuals often must live with permanent side effects resulting from chemotherapy, including hearing loss (ototoxicity) from treatment with drugs such as cisplatin. ${ }^{7}$ Cisplatininduced hearing loss is characterised by a bilateral, irreversible and high-frequency sensorineural hearing loss. The hearing loss is irreversible because once destroyed by cisplatin-based chemotherapy, mammalian hair cells cannot regenerate. ${ }^{4}$ The pathophysiology of ototoxicity has been extensively discussed in previous research, ${ }^{7,8}$ with recent evidence suggesting a hyperaccumulation of cisplatin within the cochlea as the reason behind ototoxicity. ${ }^{9}$ Incidence of cisplatin-induced ototoxicity has been reported to vary from as low as $0 \%$ to as high as $100 \%$ amongst patients in different studies. ${ }^{4}$ In one of the few South African studies on the topic, incidence of ototoxicity was reported to be $55.1 \% .^{10}$ 
There are several factors that have been reported to increase a patient's susceptibility to hearing loss owing to cisplatinbased chemotherapy. ${ }^{4,8}$ Amongst them are cumulative dosage $^{4}$ renal insufficiency, ${ }^{7}$ age, ${ }^{11,12}$ pre-existing hearing loss, ${ }^{10}$ infusion rate $^{12,13,14}$ and genetic predisposition. ${ }^{15}$ At present, it is not possible to determine, beforehand, an individual's susceptibility or risk of developing ototoxicity following treatment with cisplatin-based chemotherapy. ${ }^{13}$ Therefore, the most common strategy currently used to prevent ototoxicity owing to cisplatin-based chemotherapy is to prospectively monitor patient's hearing thresholds throughout their chemotherapy treatment, also known as ototoxicity monitoring. ${ }^{12,14,15,16,17,18}$ This allows a physician to choose from several medical interventions, once a decline in a patient's hearing sensitivity is noted, to prevent further progression in hearing loss. . $^{14,15,16}$

The medical interventions that are reportedly used to prevent further deterioration in patient's hearing thresholds during cisplatin chemotherapy are: discontinuing the administered medication, adjusting the dosage of medication, changing the frequency of drug administration, switching to a less ototoxic drug or continuing with the medication for which they would have to prepare the patient and family on coping strategies to adjust to the hearing loss. ${ }^{10,12}$ Currently, there is limited evidence on the effectiveness of these treatment modification strategies implemented post detection of deterioration in hearing, especially in the adult population. ${ }^{10}$ Use of lower cumulative cisplatin doses or less ototoxic medications such as Carboplatin has not been implemented as a standard preventative strategy for cisplatin-induced ototoxicity in clinical practice, because it is unclear whether these modifications would fully keep the antitumour efficacy. ${ }^{16}$ Nevertheless, modification strategies are often implemented as part of treatment protocols for specific cancerous tumours. ${ }^{17,18,19,20}$ For example, interventions to change the dosage and type of drug, respectively, to prevent further deterioration in hearing once it is detected have been recommended for tumours such as medulloblastoma. ${ }^{19}$

Use of cisplatin as a therapeutic agent has been found to successfully lead to remission in various cancerous tumours despite side effects such as ototoxicity. Thus, therapeutic agency often takes precedence over subsequent risk of developing ototoxicity when using this drug during chemotherapy. ${ }^{9}$ Given the high success rates of current chemotherapy treatments, many cancer survivors must live with the ototoxic side effect from mainline drugs like cisplatin. It is therefore imperative, now more than ever, to focus on measures that can be taken to ensure excellent quality of life post treatment. The current study will help to bridge the gap in literature by providing data that evaluate, in a descriptive and comparative manner, the different preventative audiological measures used when cisplatinbased ototoxicity has been detected, to determine to what extent each of them is effective in preserving patients' hearing thresholds during chemotherapeutic treatment.

\section{Methods}

A retrospective medical folder review design ${ }^{21}$ and purposive sampling were used to select medical records of all patients who underwent cisplatin-based chemotherapy in the Department of Radiation Oncology at Groote Schuur Hospital (GSH) while receiving regular ototoxicity monitoring services in the Audiology department between 2011 and 2016. Only medical folders that had at least three audiograms obtained at the start (baseline), during (checkup) and end (exit) of chemotherapy were included for review. Medical folders of patients who had a pre-existing hearing loss prior to baseline audiogram, an indication of middle ear pathology, simultaneous treatment with ototoxic medications (e.g. aminoglycosides and loop diuretics) and radiation therapy treatment for head and neck cancers were excluded from the review.

The following data were extracted from the medical records using a self-developed data abstraction sheet - demographic, medical (chemotherapy) and audiometric data. The extracted data were kept in a password-secured electronic Google spreadsheet. Anew study number, consisting of a combination of three letters and three numbers, was allocated to each folder to ensure that no patient names were recorded. Patients at this facility had their hearing status assessed prior to the start of cisplatin chemotherapy (baseline audiometry) and before each cycle of chemotherapy treatment until they completed their treatment. The following audiometric tests were used to monitor patients' hearing thresholds - otoscopy, tympanometry and pure tone audiometry $(250 \mathrm{~Hz}-8000$ $\mathrm{Hz}$ ). The Common Terminology Criteria of Adverse Events version 4 (CTCAE v4) grading scale was used to determine whether the patient's hearing thresholds were changing during chemotherapy treatment and to grade the severity of hearing loss in the current study population. ${ }^{21}$ The CTCAEv 4 is the most commonly used, valid and widely accepted hearing loss grading scale in oncology literature and clinical trials. ${ }^{21}$ It describes incidence and specifies the severity of hearing loss. It uses four grades of hearing loss which combines objective and subjective hearing assessments in rating hearing. Category zero represents no hearing loss and subsequent grades represent increasing severity of hearing loss, respectively. $22,23,24$

$R$, a statistical environment used for statistical analysis and graphics was used ${ }^{25}$ to analyse the data. Figures, graphs and tables were used to summarise, describe and arrange the data into organised visual presentations. The Chi-square test was used to assess for significant associations between categorical variables. ${ }^{26}$

\section{Ethical considerations}

Ethical clearance for the study was sought and granted by the University of Cape Town, Human Research Ethics Committee (HREC REF: 023/2017). 


\section{Results}

A total of 680 medical folders of patients who received cisplatin-based chemotherapy at GSH oncology department between 2011 and 2016 were accessed for review. Out of these, 128 folders met the inclusion criteria for this study. However, only 58 of the folders that met the inclusion criteria had information on the type and dose of chemotherapy drug used. Table 1 presents a description of patients whose folders were included in this study.

Fifty-eight patients started with cisplatin at baseline audiogram visit. At the time of the exit audiogram, this number was reduced to 44 patients. Median cisplatin cumulative dose during treatment was $465 \mathrm{mg} / \mathrm{m}^{2}$. In terms of drug dose, median single dose for cisplatin remained relatively unchanged throughout treatment (see Figure 1).

With regard to treatment modification to minimise further deterioration of hearing loss during cisplatin chemotherapy treatment, three dominant strategies were found to be used at GSH: Switching the patient to a different drug, adjusting or reducing the patient's dosage or continuing the same treatment regimen (without dosage adjustment). Figure 2

TABLE 1: Participants' description $(n=58)$.

\begin{tabular}{lcc}
\hline Patient characteristics & Frequency $(\boldsymbol{N})$ & Percentage (\%) \\
\hline Age (median, range) & $43(18-75)$ & - \\
Sex & 36 & 62.0 \\
$\quad$ Male & 22 & 38.0 \\
$\quad$ Female & & \\
Tumour type & 23 & 40.0 \\
$\quad$ Squamous cell carcinoma (SCC) & 35 & 60.0 \\
$\quad$ Other tumour types & 3 & 5.1 \\
History of noise exposure & 4 & 6.8 \\
Tinnitus & Median 13.45 & - \\
Treatment duration & (Range: 2-85) & \\
&
\end{tabular}

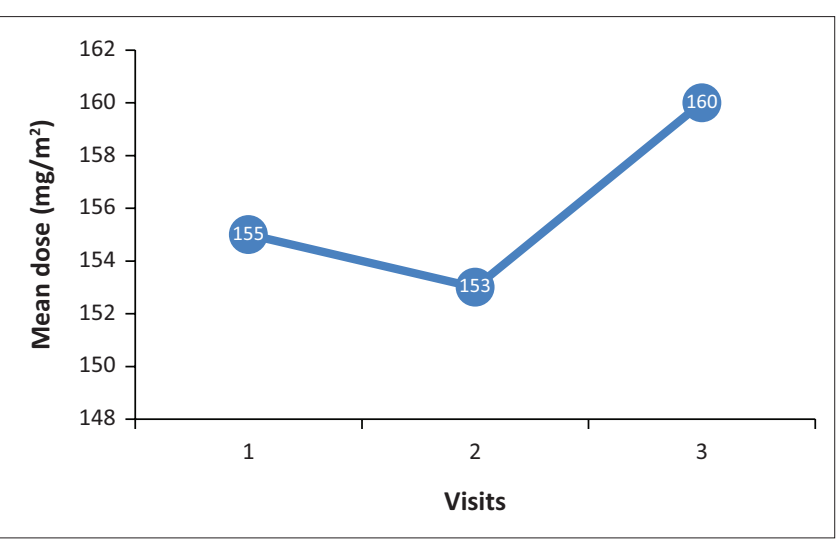

Note: Mean cisplatin dose at baseline (1), follow-up (2) and exit (3) visits. FIGURE 1: Median single cisplatin dose at different visits $(n=58)$. provides a summary of the types of strategies implemented and the proportions of patients under each one.

The CTCAEv4 criteria were used to grade presence and severity of hearing loss. Incidence of ototoxicity and severity of hearing loss according to the different treatment modifications at the end of treatment are presented in Table 2.

The Chi-square test showed no significant association between treatment modifications and incidence of hearing loss as classified by the CTCAEv 4 grading criteria $\left(\chi^{2}=2.33\right.$, $d f=2, p=0.31, p<0.05)$.

\section{Discussion}

This study set out to determine the efficacy of strategies used to prevent or minimise cisplatin-induced hearing loss in chemotherapy patients seen jointly by the departments of Oncology and Audiology as outpatients at GSH between the years 2011 and 2016. There were three common strategies that were implemented by the Department of Radiation Oncology at GSH when a patient's hearing thresholds showed signs of deterioration - adjust (reduce) the drug dosage, switch the patient to a less ototoxic drug and no modification (i.e. continue with the original treatment plan). Overall, the findings of the study indicated that despite a variety of treatment modifications being implemented when a change in patient's hearing threshold is detected, the majority of the patients continue to experience a significant deterioration in their hearing thresholds following treatment in all the noted sub-groups. The result showing no statistically significant association between treatment modifications and incidence of hearing loss was an unexpected finding in this study. This is because one of the premises upon which

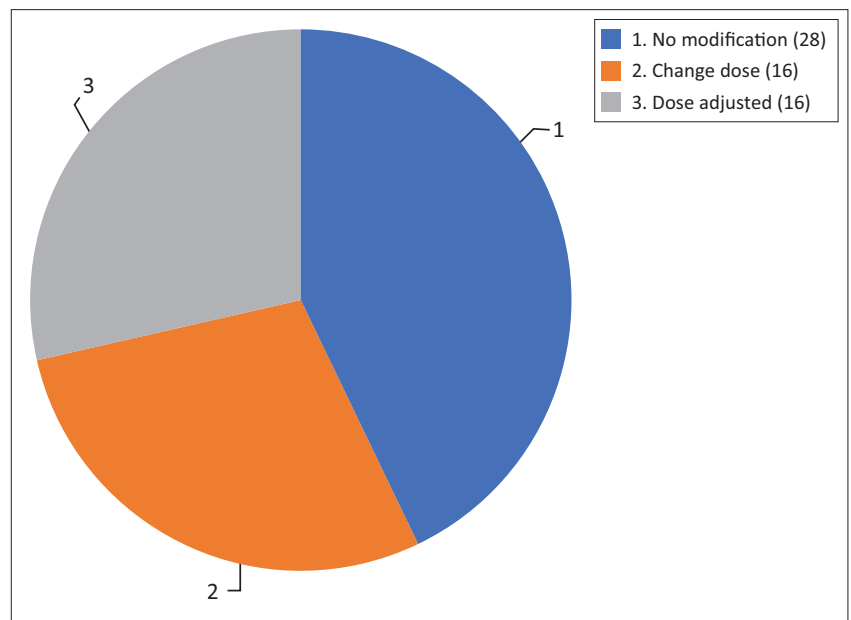

FIGURE 2: Distribution of patients according to chemotherapy treatment modification $(n=58)$.

TABLE 2: Ototoxicity and severity of hearing loss by treatment modification at the end of treatment.

\begin{tabular}{|c|c|c|c|c|c|c|c|}
\hline $\begin{array}{l}\text { Grading } \\
\text { system }\end{array}$ & Treatment modification & $N$ & Grade $0(\%)$ & Grade $1(\%)$ & Grade $2(\%)$ & Grade $3(\%)$ & Grade $4(\%)$ \\
\hline \multirow[t]{3}{*}{ CTCAEv4 } & No change & 28 & 43.8 & 9.38 & 9.38 & 18.8 & 18.8 \\
\hline & Adjusted dose & 16 & 50.0 & 6.25 & 6.25 & 12.5 & 25.0 \\
\hline & Switched drug & 16 & 25.0 & - & 18.8 & 31.3 & 25.0 \\
\hline
\end{tabular}

CTCAEv4, Common Terminology Criteria of Adverse Events version 4. 
audiological monitoring of ototoxicity in patients who are treated with ototoxic medications is the assumption that further deterioration of patient's thresholds can be minimised or prevented if an appropriate intervention is implemented. ${ }^{18,19}$

About half (50\%) of the patients whose cisplatin dose was adjusted (reduced) continued to experience a deterioration in their hearing thresholds post treatment modification. This was consistent with the findings of previous studies which reported a continuation of the deterioration of hearing thresholds post cisplatin dose adjustment or reduction. ${ }^{18,19,20}$ Therefore, while it may seem intuitive that a reduction in drug dose should lead to less deterioration of hearing thresholds owing to cisplatin-induced chemotherapy, none of the studies reviewed thus far ${ }^{18,19,20}$ and also the findings of this study seem to support that.

A possible explanation for the observed lack of effectiveness of the dose reduction strategy could be inadequacy of the audiometric test used to monitor patients' hearing thresholds to provide early warning signs when patients' hearing thresholds start to deteriorate. For instance, in this study, and all other studies reviewed which reported implementing of dose reduction to prevent further deterioration of hearing loss, ${ }^{18,19,20}$ patients' hearing thresholds were monitored using conventional pure tone audiometry $(250 \mathrm{~Hz}-8000 \mathrm{~Hz})$. This test protocol is only sensitive to ototoxic changes below or equal to $8 \mathrm{kHz}^{4}$ It is therefore highly likely that the ineffectiveness of this strategy as shown in this study, and as reported in other studies that have been reviewed thus far, was mainly owing to the fact that changes in patients' hearing thresholds were detected too late and therefore any intervention that was implemented following that was less likely to be effective. Also, all the studies reviewed that reported the ineffectiveness of the dose reduction intervention involved paediatric populations and could therefore be reflecting a ceiling effect ${ }^{26}$ owing to the reflected homogeneity in age group. Studies showing dose reductions in adult populations could not be found and therefore further research is needed which looks at this type of treatment modification in the adult population.

Another strategy that was implemented at the GSH oncology department to minimise further deterioration of patient hearing thresholds during cisplatin-based chemotherapy was to switch the patient from cisplatin to Carboplatin. Carboplatin is known to be less ototoxic than cisplatin. ${ }^{6}$ However, this strategy was also found not to be effective in preventing further deterioration of patients' hearing threshold. Patients who were managed using this strategy showed the highest incidence of ototoxicity when compared to those who were managed using other strategies. This was consistent with the findings of previous studies ${ }^{18,20}$ who also reported that this strategy was ineffective. This could potentially be because of the fact that patients who are switched from cisplatin to Carboplatin are likely to be those who are already showing susceptibility to ototoxicity. Therefore, these are patients who are likely to lose their hearing regardless of the ototoxic drug used. Therefore, switching them from cisplatin to Carboplatin which is also ototoxic is less likely to be effective. Reports from previous studies also seem to suggest that simultaneous use of ototoxic medication is a risk factor on its own. ${ }^{4,6}$ Based on the studies reviewed thus far and the findings of this study, there appears to be a synergistic effect between cisplatin and Carboplatin therapy which increases a patient's risk of developing hearing loss during chemotherapy as evidenced by the high incidence of hearing loss in that group, and it causes more severe hearing loss when compared to other treatment modification groups.

The last strategy implemented was to continue with the original treatment plan (i.e. no treatment modification). For patients for whom this strategy was implemented, the study revealed a large proportion of patients with ototoxicity at the exit stage, which was also consistent with the findings of previous studies. ${ }^{10,27,28,29}$ However, an unexpected finding from this study was that incidence of ototoxicity in patients who were managed using this strategy was comparable to that of patients who were managed using the other two strategies. It was expected that more patients who were managed using this strategy will experience a significant deterioration in hearing thresholds when compared to patients in the other two strategies; however, that was not the case. A possible explanation for this finding could be that patients who were managed using this strategy were those who showed better tolerance to cisplatin-based chemotherapy (i.e. initially showed less deterioration in hearing thresholds) and therefore were not prioritised for any treatment modification.

The findings of the current study should be interpreted while considering its methodological limitations and study design. That is, the current study was a retrospective record review, no extended high-frequency audiometry data was obtained and no audiological follow-up of patients post chemotherapy occured. Retrospective studies rely on how accurate the written records are and face difficulty when controlling for bias because there is no implementation of blinding and randomisation..$^{21}$ Also, only 58 of 680 files reviewed in this study fulfilled the inclusion criteria. This impacted on the sample size and resultantly the power of the study which enabled significant associations to be made. Lack of longterm and follow-up audiologic data meant that the current study could not assess for changes in incidence and progression of ototoxicity post treatment. Several studies have reported development of ototoxicity post treatment. ${ }^{9}$ This highlights the importance of long-term follow-up with suggestions for long-term follow periods of up to 10 years. ${ }^{11}$ The author also acknowledges that modifications to cisplatin chemotherapy treatment are not made only for the purpose of preventing ototoxicity.

However, despite its limitations, this is one of a handful of studies in South Africa that sought to overtly document and assess the effectiveness of current measures used to prevent 
further deterioration of patients' hearing thresholds during cisplatin chemotherapy. The study also revealed that conventional audiometric monitoring for ototoxicity, although widely and commonly implemented, potentially causes delays in identification of patients who are at risk of developing significant hearing loss and this could potentially render resultant treatment modifications ineffective. The outcomes from this study highlight the need for evidencebased ototoxicity prevention protocols that will ensure timeous intervention should a patient develop hearing loss while being treated using cisplatin.

\section{Conclusion}

This study revealed that current strategies implemented at Department of Radiation Oncology at GSH, namely change in dosage, change in type of drug and continuation with the same treatment regimen, were ineffective in preventing or minimising further deterioration of patients' hearing thresholds during cisplatin chemotherapy. Further research is therefore recommended to investigate the rationale behind the treatment modifications chosen in the clinical settings when ototoxicity develops as well as to try to document the effectiveness of different treatment modifications and/or intervention strategies. Findings of this study also seem to suggest that more effective and sensitive ototoxicity monitoring protocols for early detection of hearing loss in patients during cisplatin chemotherapy treatment may be more useful than conventional audiometry. More sensitive ototoxicity monitoring protocols such as the ones that include extended high-frequency audiometry $(9 \mathrm{kHz}-16 \mathrm{kHz})$ and Distortion Product Otoacoustic Emission measures should therefore be advocated for and implemented as best practice when monitoring chemotherapy patient's hearing thresholds. ${ }^{30,31}$

\section{Acknowledgements}

The authors would like to thank Groote Schuur Hospital for allowing them to conduct the research.

\section{Competing interests}

The authors declare that they have no financial or personal relationships that may have inappropriately influenced them in writing this article.

\section{Authors' contributions}

Z.C. was the lead author and L.R. was the supervisor and coauthor.

\section{References}

1. Stefan DC. Why is cancer not a priority in South Africa?. S Afr Med J. 2015;105(2):103-104. https://doi.org/10.7196/SAMJ.9301

2. Mayosi BM, Flisher AJ, Lalloo UG, Sitas F, Tollman SM, Bradshaw D. The burden of non-communicable diseases in South Africa. Lancet. 2009;374(9693):934-947. https://doi.org/10.1016/S0140-6736(09)61087-4

3. World Health Organization. 10 facts on non-communicable disease [homepage on the Internet]. Geneva; 2013 [cited 2018 Aug 03]. Available from: http://www.who. int/features/factfiles/noncommunicable_diseases/en/\#
4. Paken J, Govender CD, Pillay M, Sewram V. Cisplatin-associated ototoxicity: A review for the health professional. J Toxicol. 2016;2016:1-13

5. Waissbluth S, Peleva E, Daniel SJ. Platinum-induced ototoxicity: A review of prevailing ototoxicity criteria. Eur Arch Otorhinolaryngol. 2017;274(3):1187-1196. https://doi.org/10.1007/s00405-016-4117-z

6. Langer T, am Zehnhoff-Dinnesen A, Radtke S, Meitert J, Zolk O. Understanding platinum-induced ototoxicity. Trends Pharmacol Sci. 2013;34(8):458-469. https:// doi.org/10.1016/j.tips.2013.05.006

7. Rybak LP. Mechanisms of cisplatin ototoxicity and progress in otoprotection. Curr Opin Otolaryngol Head Neck Surg. 2007;15(5):364-369. https://doi.org/10.1097/ MOO.0b013e3282eee452

8. Rybak LP, Mukherjea D, Jajoo S, Ramkumar V. Cisplatin ototoxicity and protection: Clinical and experimental studies. Tohoku J Exp Med. 2009;219(3):177-186. https://doi.org/10.1620/tjem.219.177

9. Breglio $A M$, Rusheen $A E$, Shide $E D$, et al. Cisplatin is retained in the cochlea indefinitely following chemotherapy. Nat Commun. 2017;8(1):1654. https://doi. org/10.1038/s41467-017-01837-1

10. Whitehorn $\mathrm{H}$, Sibanda $\mathrm{M}$, Lacerda $\mathrm{M}$, et al. High prevalence of cisplatin-induced ototoxicity in Cape Town, South Africa. S Afr Med J. 2014;104(4):288-291. https:// doi.org/10.7196/SAMJ.7389

11. Dell'Aringa AH, Isaac ML, Arruda GV, et al. Audiological findings in patients treated with radio-and concomitant chemotherapy for head and neck tumors. Radiat Oncol. 2009;4(1):53. https://doi.org/10.1186/1748-717X-4-53

12. Van As JW, Van den Berg $H$, Van Dalen EC. Medical interventions for the prevention of platinum-induced hearing loss in children with cancer. Cochrane Database Syst Rev. 2014;7:CD009219.

13. Yasui N, Adachi N, Kato M, et al. Cisplatin-induced hearing loss: The need for a long-term evaluating system. J Pediatr Hematol/Oncol. 2014;36(4):e241-e245. https://doi.org/10.1097/MPH.0000000000000028

14. Yancey A, Harris MS, Egbelakin A, Gilbert J, Pisoni DB, Renbarger J. Risk factors for cisplatin-associated ototoxicity in pediatric oncology patients. Pediatr Blood Cancer. 2012;59(1):144-148. https://doi.org/10.1002/pbc.24138

15. Peters U, Preisler-Adams S, Hebeisen A, et al. Glutathione S-transferase genetic polymorphisms and individual sensitivity to the ototoxic effect of cisplatin. Anti-cancer drugs. 2000;11(8):639-643. https://doi.org/10.1097/00001813200009000-00007

16. Fausti SA, Wilmington DJ, Helt PV, Helt WJ, Konrad-Martin D. Hearing health and care: The need for improved hearing loss prevention and hearing conservation practices. J Rehabil Res Dev. 2005;42. https://doi.org/10.1682/JRRD.2005.02.0039

17. Schultz C, Goffi-Gomez MV, Liberman PH, Carvalho AL. Report on hearing loss in oncology. Brazilian journal of otorhinolaryngology. 2009;75(5):634-641. https:// doi.org/10.1016/S1808-8694(15)30510-3

18. Knight KR, Kraemer DF, Winter C, Neuwelt EA. Early changes in auditory function as a result of platinum chemotherapy: Use of extended high-frequency audiometry and evoked distortion product otoacoustic emissions. J Clin Oncol. 2007;25(10):1190-1195. https://doi.org/10.1200/JCO.2006.07.9723

19. Lafay Cousin L, Purdy E, Huang A, et al. Early cisplatin induced ototoxicity profile may predict the need for hearing support in children with medulloblastoma. Pediatr Blood Cancer. 2013;60(2):287-292. https://doi.org/10.1002/pbc.24307

20. Knight KR, Kraemer DF, Neuwelt EA. Ototoxicity in children receiving platinum chemotherapy: Underestimating a commonly occurring toxicity that may influence academic and social development. J Clin Oncol. 2005;23(34):85888596. https://doi.org/10.1200/JCO.2004.00.5355

21. Hess DR. Retrospective studies and chart reviews. Respir Care. 2004;49(10):11711174.

22. Chang KW, Chinosornvatana N. Practical grading system for evaluating cisplatin ototoxicity in children. J Clin Oncol. 2010;28(10):1788-1795. https://doi. org/10.1200/JCO.2009.24.4228

23. Theunissen EA, Dreschler WA, Latenstein MN, et al. A new grading system for ototoxicity in adults. Ann Otol Rhinol Laryngol. 2014;123(10):711-718. https:// doi.org/10.1177/0003489414534010

24. Gurney JG, Bass JK. New international society of pediatric oncology Boston ototoxicity grading scale for pediatric oncology: Still room for improvement. J Clin Oncol. 2012;30(19):2303-2306. https://doi.org/10.1200/JCO.2011.41.3187

25. R Core Team. R: A language and environment for statistical computing. Vienna, Austria: R Foundation for Statistical Computing; 2013[cited n.d.]. Available from: http://www.R-project.org/

26. Cozby P, Bates S. Methods in behavioral research. New York; McGraw-Hill; 2011.

27. Arora R, Thakur JS, Azad RK, Mohindroo NK, Sharma DR, Seam RK. Cisplatin-based chemotherapy: Add high-frequency audiometry in the regimen. Indian J Cancer. 2009;46(4):311. https://doi.org/10.4103/0019-509X.55551

28. Strumberg D, Brügge S, Korn MW, et al. Evaluation of long-term toxicity in patients after cisplatin-based chemotherapy for non-seminomatous testicular cancer. Ann Oncol. 2002;13(2):229-236. https://doi.org/10.1093/annonc/mdf058

29. Bokemeyer C, Berger CC, Hartmann JT, et al. Analysis of risk factors for cisplatininduced ototoxicity in patients with testicular cancer. Br J Cancer. 1998;77(8):1355 https://doi.org/10.1038/bjc.1998.226

30. Crundwell G, Gomersall P, Baguley DM. Ototoxicity (cochleotoxicity) classifications: A review. Int J Audiol. 2016;55(2):65-74. https://doi.org/10.3109/14992027.2015 .1094188

31. Chauhan A, Subba SH, Menezes RG, et al. Younger women are affected by breast cancer in South India-a hospital-based descriptive study. Asian Pac J Cancer Prev. 2011;12(3):709-711. 